\title{
Arrays of Metal Nanostructures Produced by Focussed Ion Beam
}

\author{
P. $\operatorname{LucheS}^{a}$, A. Di Bona ${ }^{a}$, S.F. CONTri ${ }^{a, b}$, G.C. GazzAdi ${ }^{a}$, \\ P. VAvassori ${ }^{c}$, F. Albertini ${ }^{d}$, F. CASOli ${ }^{d}$, L. NAsi $^{d}$, \\ S. FABBRICI ${ }^{d}$ AND S. VALERI ${ }^{a, b}$
}

${ }^{a}$ INFM-CNR National Research Center S ${ }^{3}$, Via Campi 213a, 41100 Modena, Italy

${ }^{b}$ Dept. of Physics, University of Modena and Reggio Emilia

Via Campi 213a, 41100 Modena, Italy

${ }^{c}$ Dept. of Physics, University of Ferrara, Via Saragat 1, 44100 Ferrara, Italy

${ }^{d}$ IMEM-CNR, Parco Area delle Scienze 37/A, 43010 Parma, Italy

We present a study of the magnetic properties of arrays of nanostructures produced in a focussed ion beam-scanning electron microscope dual beam system. The single magnetic units have been isolated either by direct removal of parts of the metallic film or by local modification of the film magnetic properties. The final quality of the shape and the residual damage strictly depend on beam parameters (spot size and pixel dwell time) and on the swelling properties of the patterned materials. On square $\mathrm{Fe}(001)$ elements with a well-defined intrinsic (magnetocristalline) and shape- and size-induced (shape plus configurational) anisotropy we show that the overall magnetic anisotropy is not a mere superposition of the individual contributions. We also demonstrate that with ion irradiation doses below the milling threshold $\mathrm{L} 1_{0}$ FePt films with perpendicular magnetic anisotropy undergo a transition from the magnetically hard $\mathrm{L} 1_{0}$ phase to the magnetically soft $\mathrm{A} 1$ phase leading to an out-of-plane to in-plane spin reorientation. The magnetic properties of the planar arrays obtained by local modification of the film are compared to arrays of sculpted structures of the same material.

PACS numbers: 75.30.Gw, 75.50.Bb, 75.75.+a, 78.20.Ls, 68.49.Sf

\section{Introduction}

Micro/nano sized magnetic elements can exhibit magnetic properties different from the corresponding bulk-like or film-like phases, because the sample size becomes comparable to the intrinsic magnetic length scales (e.g. exchange length or domain wall thickness). The new properties which come into play are very interesting for both basic and applied research [1]. The fabrication of ultra 
small nanostructures of magnetic materials of increasing overall quality is therefore a strong scientific and technological challenge. Typically the structures are arranged into ordered arrays on which both the individual and collective behavior of the elements can be probed. Technologically, ordered magnetic patterns are important in applications such as magnetic random access memory (MRAM), patterned recording media, magnetic switches, magnetic logic devices, etc. [2-5]. These applications require a high degree of control on the quality of the magnetic material and on the geometry and morphology of the arrays. Focused ion beam (FIB) is a versatile nanofabrication tool based on the interaction of nanosized beams of energetic $\mathrm{Ga}^{+}$ions with solids [6-8]. With respect to state-of-the-art lithographic technologies, FIB offers a comparable resolution (a few tens of nm) with higher flexibility, enabling one-step maskless etching. Etching occurs by physical ion sputtering, optionally gas-assisted to enhance material removal rates or species selectivity [9].

In this article, we report our recent investigations in FIB-prepared, ordered magnetic nanostructures. Section 2 is dedicated to a short review of FIB preparation of magnetic structures. In Sect. 3 we present a systematic study of the dependence on the FIB parameters of the surface morphology and geometric shape of square $\mathrm{Fe} / \mathrm{NiO}$ and $\mathrm{Fe}$ elements on $\mathrm{MgO}(001)$. The magnetic properties of these structures are discussed in Sect. 4, with emphasis on magnetocrystalline vs. configurational anisotropy. Finally, in Sect. 5 we describe a study of the interaction of $\mathrm{Ga}^{+}$ions with $\mathrm{L} 1_{0} \mathrm{FePt}$ films with perpendicular anisotropy and of the properties of planar arrays of perpendicular structures.

\section{FIB fabricated magnetic nanostructures}

In a typical FIB apparatus, a beam of ions (usually $\mathrm{Ga}^{+}$ions) produced in a liquid-metal ion source, is extracted and focused by a series of electrodes, electron lenses and mechanical apertures into a beam with a diameter of a few nanometers in size. The beam is scanned by an electrostatic deflection system across an "exposure field" that, depending on the desired pixel resolution, can range between a few to hundreds of $\mu \mathrm{m}$, while a precision motion stage allows the stepping of the exposure field across the entire substrate. Any pattern can thus be milled directly on the substrate (direct writing), without the need of resist or masks. The ion energy can be varied in the $5-30 \mathrm{keV}$ range and the current intensity, which is proportional to the beam spot size, can be chosen between a few pA (a smaller spot size) to several nA (a larger spot size). In several commercially available apparatuses the FIB column operates at normal incidence and it is associated with an oblique incidence scanning electron microscope (SEM) beam in the so-called dual beam systems, allowing a simultaneous high resolution, non-destructive, analysis of the produced structures. On the other hand, like for e-beam lithography, the sequential character of FIB milling is a slow process if compared to a standard lithographic nanofabrication. 
FIB has been proved to be effective in isolating single magnetic units either by inducing localized damage $[10,11]$ or by direct removal of parts of a thin film $[12,13]$. Properties like magnetization switching and magnetic anisotropy have been measured either on the individual nanomagnets, using local magnetic probes [14, 15], or collectively, using integral probes that measure average magnetic quantities [16]. Combining perpendicular magnetization media and FIB milling to produce artificial grains in the magnetic film through the cut of trenches or grooves, storage densities as high as $200 \mathrm{Gbit} / \mathrm{in}^{2}$ have been achieved [17]. The size and the shape of the magnetic bit, which can be precisely trimmed by the FIB process, have been optimized and tested in simulated working conditions (spin stand tester) [18].

Ion irradiation at doses below the milling threshold has also been shown to induce processes such as intermixing in multilayered films, chemical or structural ordering or disordering in crystalline materials, diluting exchange interaction, production of defects or pinning centers, which result in a change of the magnetic properties of the material. The irradiation of $\mathrm{Co} / \mathrm{Pt}$ and $\mathrm{Fe} / \mathrm{Pt}$ multilayers by $\mathrm{He}^{+}$ions has been found to modify the magnetic properties (e.g. coercivity, Curie temperature, anisotropy) of the film in a highly controlled manner [19-22]. FePt and CoPt films have been found to have a better or a worse chemical order, and thus a higher [23-25] or a lower magnetic anisotropy [26], after the irradiation with light ions $\left(\mathrm{He}^{+}\right.$and $\left.\mathrm{B}^{+}\right)$depending on the used dose. The production of magnetic patterns on continuous magnetic films without significant modification of the surface roughness or of the film optical indices has been demonstrated by parallel irradiation through lithographically patterned stencil masks [19], but the results have also been reported by localized delivery of the damage, using FIB facilities [10, 27, 28].

Direct-write of materials in the nanometer scale can be also achieved through ion beam induced deposition (IBID). A precursor gas containing the material of interest (usually an organo-metallic compound) is dissociated by the FIB. The volatile species leave the surface and are exhausted out of the system, while the metal atoms are deposited on the surface and linewidths as small as a few tens of $\mathrm{nm}$ can be written. FIB ion induced deposition of $\mathrm{Co}, \mathrm{FePt}$, and CoPt arrays of circular particles with $\sim 2 \mu \mathrm{m}$ diameter and 100-200 $\mathrm{nm}$ height has been demonstrated [29, 30].

\section{Morphology of FIB fabricated regular arrays of submicrometric magnetic particles}

In this section we report on the fabrication of submicron-scaled patterns from $\mathrm{Fe}$ layers and $\mathrm{Fe} / \mathrm{NiO}$ bilayers epitaxially grown in UHV on $\mathrm{MgO}(001)$ substrates and capped with a $10 \mathrm{~nm} \mathrm{MgO}$ layer to prevent Fe oxidation. Arrays of 500 to $250 \mathrm{~nm}$ square elements with different spacing/size ratios were patterned in a FIB-SEM dual beam system by $30 \mathrm{keV}$ Ga ion beam erosion of the films. Parameters like beam spot size and pixel dwell time (DT), playing an important role 
in determining the final shape of the isolated features [31, 32], were systematically varied [33, 34]. The surface morphology and overall shape of the features were studied by in situ high-resolution SEM, and by ex situ atomic force microscopy (AFM) operating in contact mode.

An $\mathrm{MgO}(001)$ single crystal substrate was chosen since its lattice parameter is suitable for epitaxial growth of $\mathrm{Fe}$ and $\mathrm{NiO}(1.4 \%$ and $3.2 \%$ lattice mismatch, respectively). The films were prepared in a UHV molecular beam epitaxy (MBE) system (a base pressure lower than $5 \times 10^{-11}$ Torr), on ex situ cleaved substrates. $\mathrm{NiO}$ films were prepared on the $\mathrm{MgO}$ substrate at $520 \mathrm{~K}$ by depositing $\mathrm{Ni}$ from a Knudsen cell in a background oxygen pressure of $1 \times 10^{-7}$ Torr $[35,36]$. Fe films were deposited at RT from a Knudsen cell on top of predeposited NiO film or directly on the $\mathrm{MgO}$ substrate [37-39]. Finally the samples were capped with $10 \mathrm{~nm} \mathrm{MgO}$ films to protect the Fe film surface with a transparent material, suitable for magneto-optical characterization. A chemical analysis of the grown layers was performed by X-ray photoelectron spectroscopy (XPS) and their crystalline quality was monitored by photoelectron diffraction (PD) [37-39].

The samples were milled to a depth sufficient to completely remove the $\mathrm{MgO} / \mathrm{Fe}$ bilayer or the $\mathrm{MgO} / \mathrm{Fe} / \mathrm{NiO}$ try-layer, using a fixed ion dose of $2.3 \times$ $10^{17}$ ions $/ \mathrm{cm}^{2}$. Figure 1 shows 3D AFM images of arrays of square Fe dots of $500 \mathrm{~nm}$ size, obtained by milling the surface of $10 \mathrm{~nm} \mathrm{MgO} \mathrm{/} 10 \mathrm{~nm} \mathrm{Fe} \mathrm{/} \mathrm{MgO}$ sample with a beam current of $150 \mathrm{pA}$ and $420 \mathrm{pA}$ and DT of $10 \mu \mathrm{s}$, respectively. SEM images of single squares and AFM line profiles taken along the FIB milling direction are also shown in Fig. 1. All dots are characterized by a square shape with decreasing size and increasing edge roundness as the beam current grows. The most characteristic feature common to all magnetic submicron squared dots

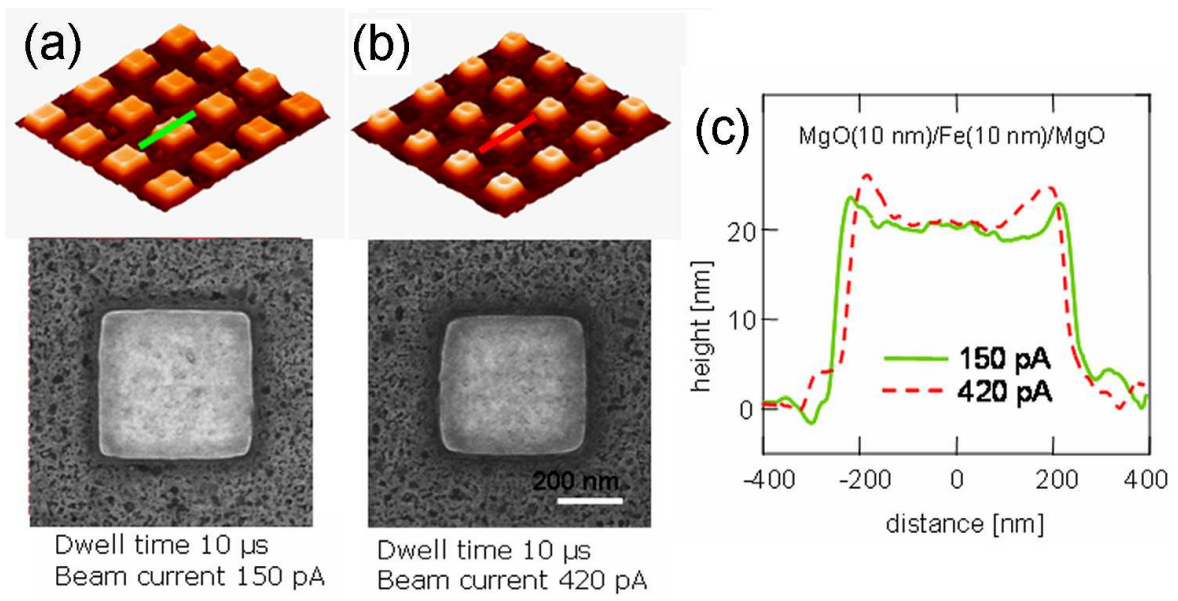

Fig. 1. (a) AFM 3D images of FIB patterns, and (b) SEM views of individual dots, for $I=150 \mathrm{pA}$ and $I=420 \mathrm{pA}$, respectively, on a $\mathrm{MgO}(10 \mathrm{~nm}) / \mathrm{Fe}(10 \mathrm{~nm}) / \mathrm{MgO}(001)$ sample; (c) corresponding line profiles on the individual dots. 
is the concave surface. Line profiles confirm that the dot width decreases from the nominal value with increasing current and show that the flat region on the dot surface is reduced in the highest current curve. The average height of the edge maxima with respect to the center of the island monotonically decreases as DT increases (not shown here). Similar results were obtained by patterning the $\mathrm{MgO} / \mathrm{Fe} / \mathrm{NiO}$ trilayer on $\mathrm{MgO}$ [33]. The overall shape of individual structures sculpted by FIB has been reported to originate in the interplay between swelling and milling processes [33, 34, 40-42]. At the early stages of ion irradiation, a relevant swelling effect has been observed in several crystalline materials like $\mathrm{Si}$, $\mathrm{Ge}, \mathrm{SiC}$, with protrusion of the irradiated area. The effect is ascribed to ion-induced amorphization of the crystalline structure, causing a local decrease of the material density and a consequent volume expansion at the surface, and/or to ion implantation in the crystalline structure of the bombarded material. As the ion dose increases, the milling process prevails and the sputtering erosion takes place.
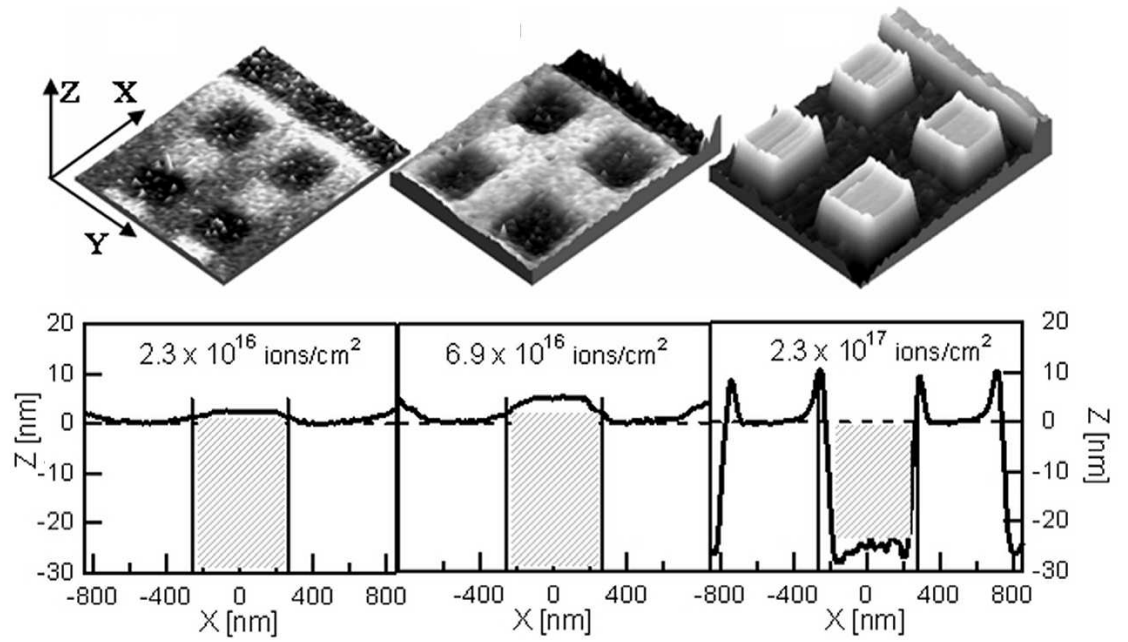

Fig. 2. AFM 3D images and corresponding line profiles for FIB patterns irradiated with increasing ion dose. The FIB-irradiated region is cross-hatched.

To clarify the role of swelling in determining the shape of the individual, multilayer magnetic elements, we irradiated the surface of a $\mathrm{MgO}(10 \mathrm{~nm}) / \mathrm{Fe}$ $(10 \mathrm{~nm}) / \mathrm{NiO}(10 \mathrm{~nm}) / \mathrm{MgO}$ (bulk) sample to obtain arrays of $500 \mathrm{~nm}$ square dots. The focused ion beam was operated at $150 \mathrm{pA}$ beam current, $1 \mu \mathrm{s}$ dwell time, and the ion dose was varied between $2.3 \times 10^{16}$ and $2.3 \times 10^{17} \mathrm{ions} / \mathrm{cm}^{2}$. The AFM 3D images and the line profiles of the resulting patterns are shown in Fig. 2. At the lowest dose the onset of surface swelling can be observed in the irradiated regions. Increasing the ion dose by a factor three results in an enhancement of the swelling. The irradiation at the highest dose produces the expected surface erosion with square islands having the surface edge bending, as already observed in the $\mathrm{MgO} / \mathrm{Fe}$ bilayer dots. From the evolution of the line profiles with increasing ion 
dose in the region around the island edge we can ascribe the edge bending effect to swelling due to the irradiation from the tails of the beam. In order to identify the swelling contribution of the individual layers to the observed shape of the multilayer dots, the same experiment was repeated on an ex situ cleaved $\mathrm{NiO}$ single crystal (001) surface, and on an $\mathrm{Fe}(001)$ single crystal prepared with the usual sputtering/annealing procedure. AFM measurements on the irradiated regions reveal that a not negligible but very weak swelling occurs in $\mathrm{NiO}$, resulting in a maximum of $1 \mathrm{~nm}$ surface protrusion at an ion dose of $5 \times 10^{15} \mathrm{ions} / \mathrm{cm}^{2}$. At larger ion doses surface milling becomes dominant. Ion irradiation does not induce any detectable swelling of the $\mathrm{Fe}(001)$ single crystal surface, whose morphology appears unchanged up to an ion dose of $3 \times 10^{16}$ ions $/ \mathrm{cm}^{2}$, when surface milling becomes evident. These results exclude any significant $\mathrm{Fe}$ and $\mathrm{NiO}$ contribution to the relevant swelling effect observed in the $\mathrm{MgO} / \mathrm{Fe} / \mathrm{MgO}$ system. It can be therefore concluded that in this system the volume expansion responsible for the overall final shape of the individual magnetic dots originates in $\mathrm{MgO}$, and specifically in the $\mathrm{MgO}$ substrate. In fact (i) the maximum in the ion-induced, in-depth damage distribution calculated by TRIM program [43] occurs at a depth that corresponds to the substrate, (ii) the measured swelling is nearly independent on the thickness of the capping layer, and (iii) in the case of a very thin capping layer $(5 \mathrm{~nm})$ the measured swelling is close to the maximum protrusion height $(6 \mathrm{~nm})$ and this would imply a non realistic reduction of the $\mathrm{MgO}$ volume density of about a factor 2 . The swelling process is generally ascribed to lattice disordering or defects accumulation rather than to ion implantation, whose contribution is considered to be negligible $[40,41]$. To clarify this point in our samples we performed a $1 \mathrm{keV} \mathrm{Ar}^{+}$sputter assisted in-depth Auger analysis on a $5 \mathrm{~nm} \mathrm{MgO} \mathrm{/} 10 \mathrm{~nm}$ Fe / MgO(001) sample previously irradiated with $5 \times 10^{16}$ ions $/ \mathrm{cm}^{2}$, an ion dose that corresponds to the maximum swelling. From the elemental depth-profile (not reported here) the average Ga concentration over a depth of $20 \mathrm{~nm}$ below the surface was estimated to be about $3 \%$. This Ga concentration value is expected to induce a swelling of about $0.3 \mathrm{~nm}$, a value that does not account for the measured $6 \mathrm{~nm}$ swelling. We then conclude that swelling should be related to defect accumulation on the $\mathrm{MgO}$ substrate, as already observed on other materials [40, 41].

The amorphization and possibly the intermixing, associated to the swelling phenomenon, are expected to reduce the interface sharpness of the ferromagnetic/antiferromagnetic bilayer over a considerable portion of the nanomagnet area. The exchange bias effect is larger in the presence of a sharp ferromagnetic/antiferromagnetic interface [44], therefore this partial interfacial broadening is in turn expected to reduce the exchange effect. In addition, the localization of damage at the edges of the nanostructures is expected to affect the magnetostatic dipolar (shape and configurational) contribution $[16,45]$ and the overall anisotropy of the magnetic structure. 


\section{Magnetic anisotropies in squared Fe micromagnets}

The anisotropy of small magnetic elements can be controlled to a large extent by imposing a suitable shape to the magnets. This allows the engineering of the so-called "shape anisotropy" [46], which for a non-uniform magnetization state conventionally includes the "true" shape anisotropy (i.e. the anisotropy of the magnet in the uniform magnetization state) and a correction term called "configurational anisotropy" [45, 47, 48], which accounts for the energy difference between the uniform and the actual magnetization state of the magnet. In general, the configurational term is often hidden by the large value of the shape anisotropy, but it becomes the dominating "shape-dependent" term in highly symmetric magnets, like discs or squares, where the shape anisotropy is exactly zero in the plane of the magnet. The magnetic material may also have intrinsic magnetocrystalline anisotropy. This contribution, together with the shape and configurational contributions, determines the total anisotropy of the magnet. Considering that the magnetic properties of an element depend critically on its anisotropy, the understanding and control of the overall anisotropy in nanomagnets is essential.

To study the interplay between magnetocrystalline and configurational anisotropies, we fabricated by FIB milling a set of arrays of Fe single crystal square elements on $\mathrm{MgO}[16,49]$. In this system the intrinsic magnetocrystalline anisotropy of the Fe single crystal has a fourfold symmetry, which is the same main symmetry showed by the configurational anisotropy in square nanomagnets [45, 47, 48]. Single crystal, $10 \mathrm{~nm}$ thick Fe films have been grown on ex situ cleaved $\mathrm{MgO}(001)$ substrates by MBE and capped with a $10 \mathrm{~nm}$ thick $\mathrm{MgO}$ layer to avoid oxidation. A FIB operating with $30 \mathrm{keV} \mathrm{Ga}^{+}$ions has been used to selectively remove portions of the film to produce the different arrays (the area of each array is $150 \times 150 \mu \mathrm{m}^{2}$ ). The FIB was combined with a field-emission SEM (dual beam system), enabling in situ high-resolution imaging of the patterned arrays.

The samples were magnetically characterized with a $\mu \mathrm{MOKE}$ setup for measuring the longitudinal Kerr effect, focusing the laser beam over a circular spot with diameter of about $10 \mu \mathrm{m}$. The longitudinal $\mu \mathrm{MOKE}$ measurements are carried out using the modulation polarization technique (modulation frequency of $50 \mathrm{kHz}$ ) because of its high signal to noise ratio. The same setup was used for modulated-field magneto-optical anisometry (MFMA) measurements [50]. This technique involves the application of two mutually orthogonal in-plane magnetic fields to the sample. In detail, a large static transversal field $H_{\mathrm{DC}}$ is applied in the sample plane perpendicularly to the direction of magneto-optical sensitivity. A small (35 Oe) longitudinal oscillating field $H_{\mathrm{AC}}$ is applied in the direction of magneto-optical sensitivity, in order to force the magnetization to oscillate around $H_{\mathrm{DC}}$. The measured response, $m_{x}(t)$, is proportional to the transverse susceptibility $\chi_{t}$ of the sample and allows us to determine the strength and the symmetry of the magnetic anisotropy [50]. 

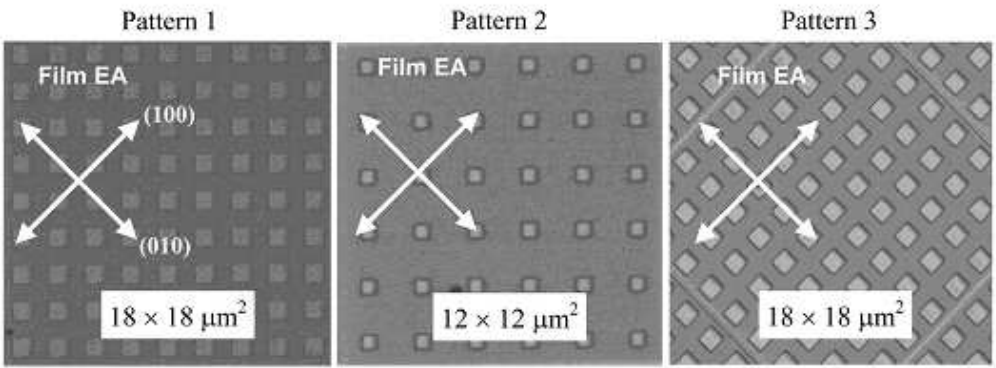

Fig. 3. SEM images of portions of the patterned areas. The white arrows indicate the direction of the magnetocrystalline easy axes of the film. Reprinted from [16] with permission.

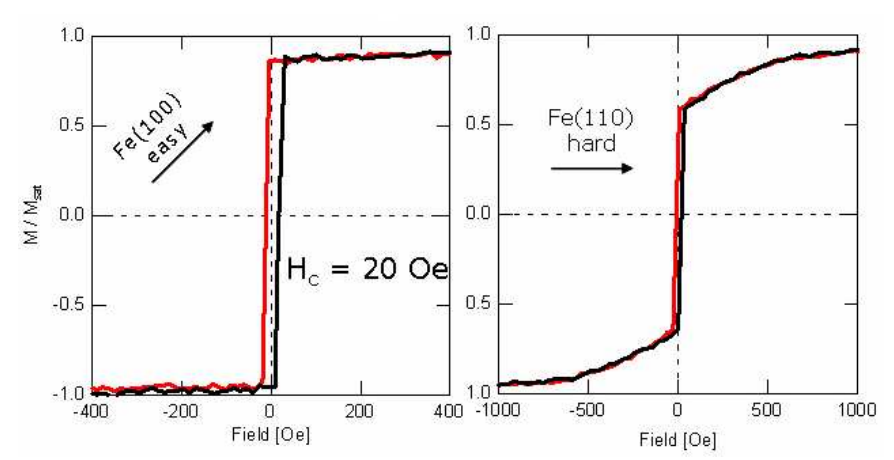

Fig. 4. $\mu \mathrm{MOKE}$ hysteresis loops with the field applied along the easy (left graph) and hard (right graph) axis of the Fe film. $M / M_{\text {sat }}$ is ratio between magnetization and saturation magnetization.

Figure 3 shows SEM images of portions of three samples. Pattern 1 is an array of square elements of $1 \mu \mathrm{m}$ side, separated by $1 \mu \mathrm{m}$; in pattern 2 the lateral size of the square elements is reduced to $500 \mathrm{~nm}$; pattern 3 is an array of square elements of $1 \mu \mathrm{m}$ side in-plane rotated by $45^{\circ}$ with respect to pattern 1 , i.e. with the sides of the squares parallel to Fe easy axis as indicated in Fig. 3. The interelement distance has been chosen to be large enough that magnetostatic interactions between the nanomagnets are negligible compared to the other energy contributions [51].

The easy and hard axis $\mu$ MOKE loops of the continuous Fe film, taken in the proximity of the patterns, are shown in Fig. 4. No out-of-plane component of the magnetization has been found, as expected for such thick Fe layers. It is worth noting that the small coercive field $(\sim 20$ Oe to be compared with the hard axis saturation field larger than $500 \mathrm{Oe}$ ) displayed by the easy axis loop indicates that the magnetization reversal is determined by nucleation and expansion of reversed 

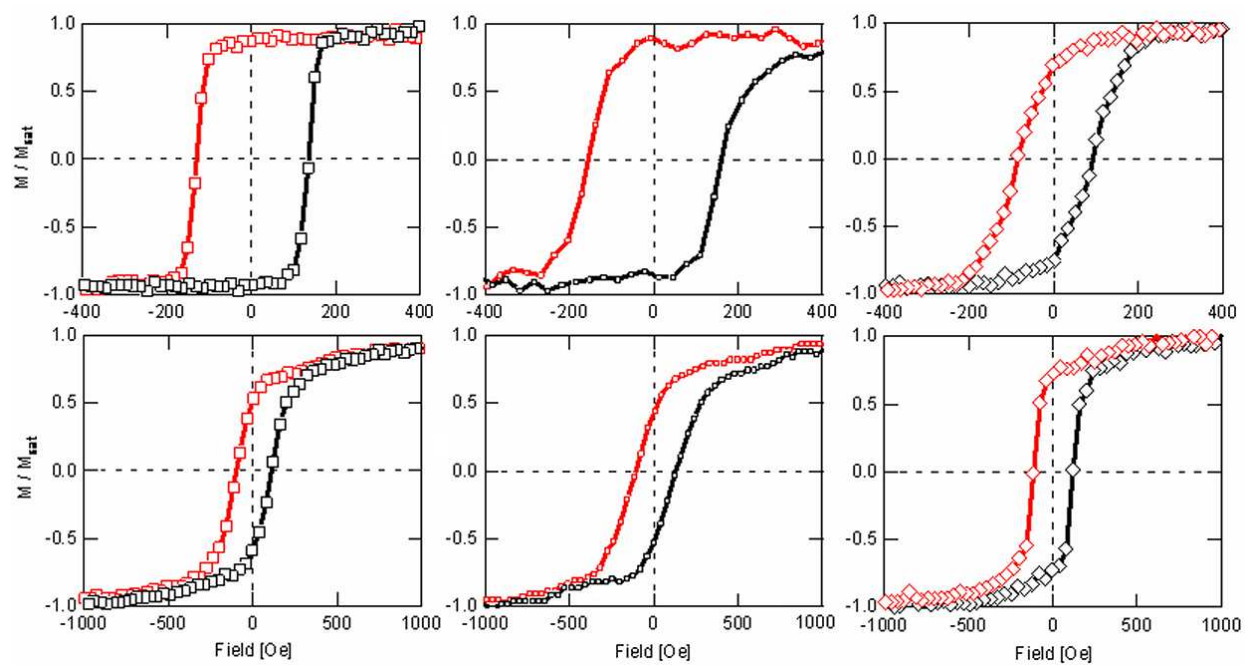

Fig. 5. $\mu \mathrm{MOKE}$ easy (top part) and hard (bottom part) axis hysteresis loops of patterns 1, 2, and 3 in Fig. 3.

domains, as already observed for thin single crystal Fe films [52]. The hysteresis loops of the patterned structures, taken for $H$ applied along the easy and hard directions of the film, are shown in Fig. 5. The shape of the loops evidences that patterns 1 and 2 have the same easy and hard magnetization directions as the film, while for pattern 3 we observe a substantial reduction of the ratio between remnant and saturation magnetization, $M_{\mathrm{r}} / M_{\mathrm{s}}$, in the loop with $H$ parallel to Fe [100] direction $\left(M_{\mathrm{r}} / M_{\mathrm{s}}=0.74\right.$ compared to 0.9 for the other two patterns) and the opposite behavior in the loop with $H$ parallel to Fe [110] direction $\left(M_{\mathrm{r}} / M_{\mathrm{s}}=0.67\right.$ compared to $\sim 0.5$ ). The different loop shapes can be understood considering the relative orientation of the film easy axes and the patterned structures as shown by the white arrows in the SEM image of Fig. 3. The square elements of patterns 1 and 2 have been oriented in a way to have their diagonals parallel to the film easy axes, while in pattern 3 the squares have their diagonal parallel to the film hard axes. At first order, the configurational anisotropy in square nanomagnets of this size and the thickness was found to have in-plane fourfold symmetry with easy directions along the square diagonals [45]. Being the easy axis directions of configurational anisotropy in patterns 1 and 2 coincident with those of intrinsic magnetocrystalline anisotropy, the symmetry of the overall anisotropy of the square nanomagnets is expected to be the same as in the continuous film. For pattern 3, the easy and hard directions of crystalline and configurational anisotropy are competing explaining the "less easy" and "less hard" shapes of the loops. The hysteresis loops of the patterned areas have a coercive field much larger than the continuous film. These differences, confirmed also by micromagnetic simulations [16], are determined by the lateral confinement, which hinders the formation of domains during the mag- 
netization reversal. The higher energy barrier for domain nucleation is determined by the balance between the reduction of the internal magnetostatic energy resulting from the breaking up into domains and the energy increase required to set up domain walls. As a result, the nucleation of magnetization reversal is retarded, the coercive field increased compared to the continuous film and the magnetization switching takes place more gradually.

The anisotropy symmetry is obtained by detecting the angular dependence of the MFMA signal with the transversal field set at a value large enough to avoid the formation of domains. In Fig. 6 we show the polar plot of $1 / \chi_{t, H_{0}}(\theta)$, with $H_{0}=700$ Oe, measured from the three patterns. The plots relative to patterns 1 and 2 show a fourfold symmetry, unvaried with respect to the film, as expected being the easy axis directions of configurational anisotropy coincident with those of intrinsic magnetocrystalline anisotropy. The differences in the width of the
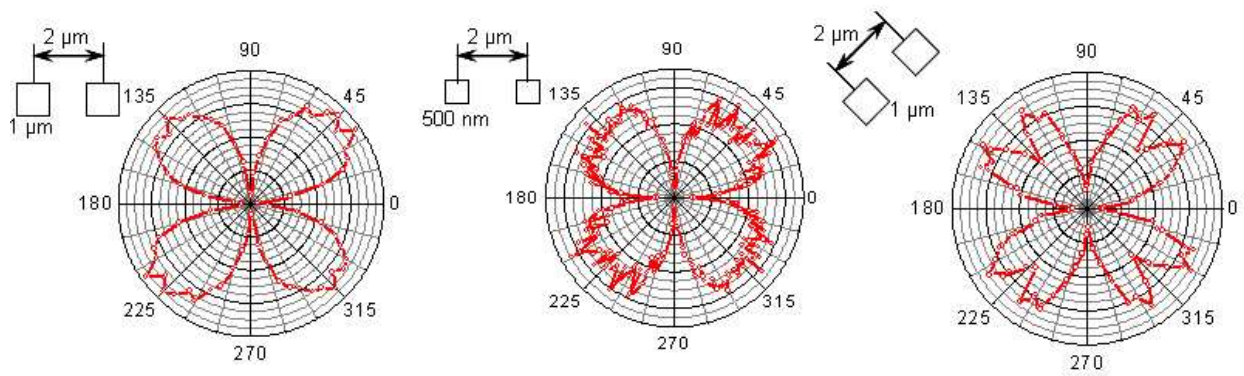

Fig. 6. Polar plots of $1 / \chi_{t}$ as a function of the applied field direction measured from patterns 1, 2, and 3 in Fig. 3. The angle is measured from the film hard axis [110] direction. The amplitudes of the fixed external field and of the transverse oscillating field are 700 Oe and 35 Oe, respectively.

lobes in the plots of the two patterns are ascribed to higher-order effects [16]. The polar plot of pattern 3 (on the right side of Fig. 6) displays a clear eightfold anisotropy with sharp minima around $n \pi / 4$. The shapes and symmetries of the $1 / \chi_{t, H_{0}}(\theta)$ plots have been reproduced by micromagnetic simulations [16]. The explanation for this dominating eightfold symmetry in the case of pattern 3 is not trivial. First of all it should be noted that, in this case, being the sides of the squares (i.e. the configurational hard axes) aligned with the magnetocrystalline easy axes, the superposition of the magnetocrystalline and first order configurational anisotropies should result in a reduced overall fourfold anisotropy (vanishing if the two contributions were of equal strength). The observed eightfold symmetry has been ascribed to a small fourfold anisotropy, resulting from the partial cancellation of the magnetocrystalline and configurational fourfold terms, plus a higher order eightfold-symmetric term of the configurational contribution [16]. 


\section{Local modification of the magnetic properties by $\mathrm{Ga}^{+}$ions irradiation}

$\mathrm{L} 1_{0} \mathrm{FePt}$ films with perpendicular anisotropy have been chosen for the study of the modification of magnetic properties by ion irradiation. An $\mathrm{MgO}(100)$ substrate has been chosen in order to obtain the epitaxial $\mathrm{L} 1_{0}$ phase with the $c$ axis, and as a consequence the magnetic anisotropy, oriented perpendicular to the surface. Films of $10 \mathrm{~nm}$ thickness were grown in a RF sputtering apparatus by alternate deposition of about $0.2 \mathrm{~nm}$ Fe and Pt layers. The substrate was kept at $T=400^{\circ} \mathrm{C}$ in order to promote a high degree of ordering and high values of perpendicular squareness [53].

The film structural and morphological characterization has been performed by XRD, AFM, and scanning tunneling microscopy (STM) [53, 54]. Alternating gradient force magnetometry (AGFM) in parallel and perpendicular configuration, $\mu \mathrm{MOKE}$ in longitudinal and polar geometry and magnetic force microscopy (MFM) have been used for the magnetic characterization. As a first step we
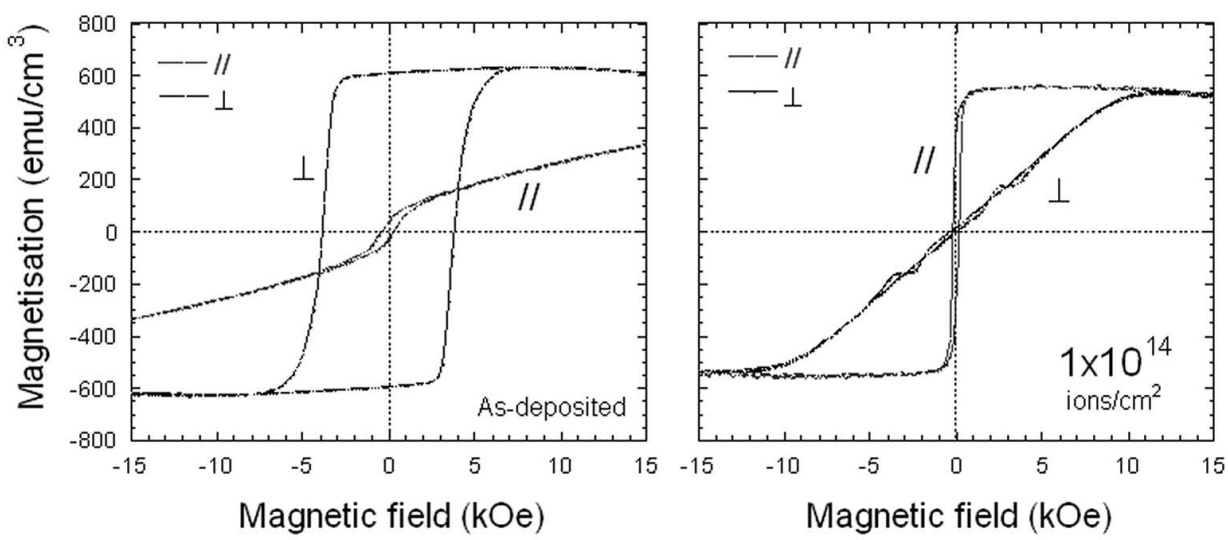

Fig. 7. AGFM hysteresis cycles measured with the magnetic field parallel (black line) and perpendicular (red or gray line) to the film plane before irradiation (left) and after irradiation at the lowest effective ion dose $\left(1 \times 10^{14}\right.$ ions $\left./ \mathrm{cm}^{2}\right)$ (right).

studied the films after uniform irradiation with $\mathrm{Ga}^{+}$ions at doses ranging from $1 \times 10^{13}$ to $4 \times 10^{16}$ ions $/ \mathrm{cm}^{2}$. TRIM simulations [43] have allowed us to calculate the optimum ion energy to be used in order to minimize the presence of implanted $\mathrm{Ga}^{+}$ions within the film. The maximum energy available on our FIB apparatus, $30 \mathrm{keV}$, was used. XRD data (not shown here) indicated that, for $\mathrm{Ga}^{+}$ doses ranging from $1 \times 10^{14}$ to $1 \times 10^{16}$ ions $/ \mathrm{cm}^{2}$, a complete transition from the ordered $\mathrm{L} 1_{0}$ to the disordered A1 structure takes place. At $5 \times 10^{13}$ ions $/ \mathrm{cm}^{2}$ a significant fraction of $\mathrm{L} 1_{0}$ phase is still present, whereas for doses larger than $1 \times 10^{16}$ ions $/ \mathrm{cm}^{2}$ film erosion becomes relevant. Figure 7 shows AGFM hysteresis cycles measured with the magnetic field parallel and perpendicular to the film 
plane before the irradiation (left) and after the irradiation at the lowest effective ion dose $\left(1 \times 10^{14}\right.$ ions $\left./ \mathrm{cm}^{2}\right)$ (right). The disordering from face centered tetragonal $\mathrm{L} 1_{0}$ to cubic $\mathrm{A} 1$ phase eliminates the perpendicular magnetocrystalline anisotropy that arises from the tetragonal structure made of alternating pure $\mathrm{Pt}$ and Fe layers. As a consequence the perpendicular coercivity is suppressed. It is also worth noticing that the drop of perpendicular anisotropy produces a spin-reorientation-transition corresponding to the change of easy-magnetization-direction from the perpendicular [001] direction to the in-plane direction. The MFM signal, which is sensitive to perpendicular field gradients, drops to zero.

We have also studied the changes of morphology induced by ion irradiation of FePt films. The SEM image in Fig. 8, shows a close-up view of $1 \mu \mathrm{m}$ diameter dots, obtained by irradiating the surroundings of the dots with $1 \times 10^{14}$ ions $/ \mathrm{cm}^{2}$ ion dose. The morphology of the films (appearing darker than the dot in Fig. 8) shows the same maze-like interconnected grains structure of non irradiated dots, with no evidence of swelling effect.

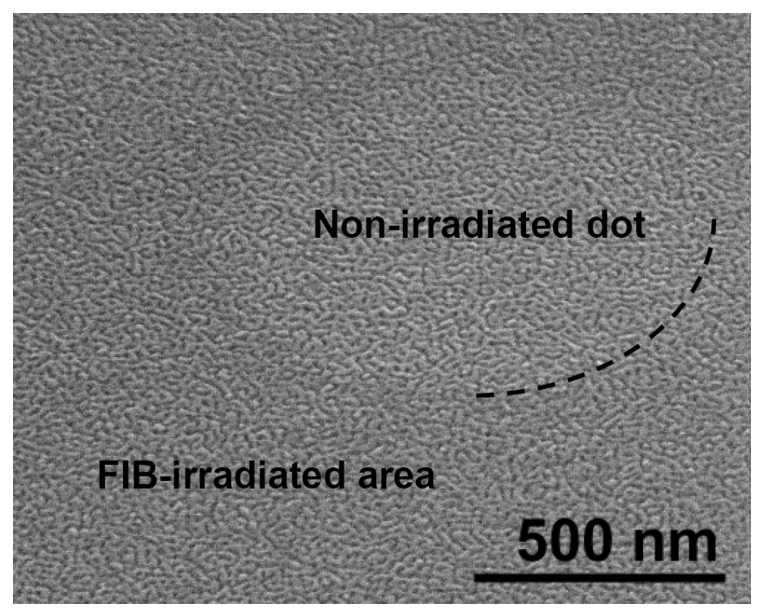

Fig. 8. SEM image of a portion of a FePt film in which a non irradiated $1 \mu$ m diameter dot is surrounded by areas irradiated by $\mathrm{Ga}^{+}$ions at a dose of $1 \times 10^{14}$ ions $/ \mathrm{cm}^{2}$. A dashed line marks one quarter of the dot border. The dot appears elliptical because the sample is tilted by $52^{\circ}$.

By using the lowest effective dose $\left(1 \times 10^{14} \mathrm{ions} / \mathrm{cm}^{2}\right)$, two-dimensional continuous patterns were fabricated, without modifying the surface topography. In particular, two patterns composed by hard magnetic $\mathrm{L} 1_{0}$ and soft $\mathrm{A} 1$ phases were produced: non irradiated dots of $1 \mu \mathrm{m}$ and $250 \mathrm{~nm}$ diameter, separated by irradiated areas with a spacing between the structures equal to their diameter. In order to compare their properties with the corresponding physically isolated nanostructures we have also produced patterns of $1 \mu \mathrm{m}$ diameter dots by using an ion dose above the milling threshold $\left(2.6 \times 10^{16}\right.$ ions $\left./ \mathrm{cm}^{2}\right)$. 

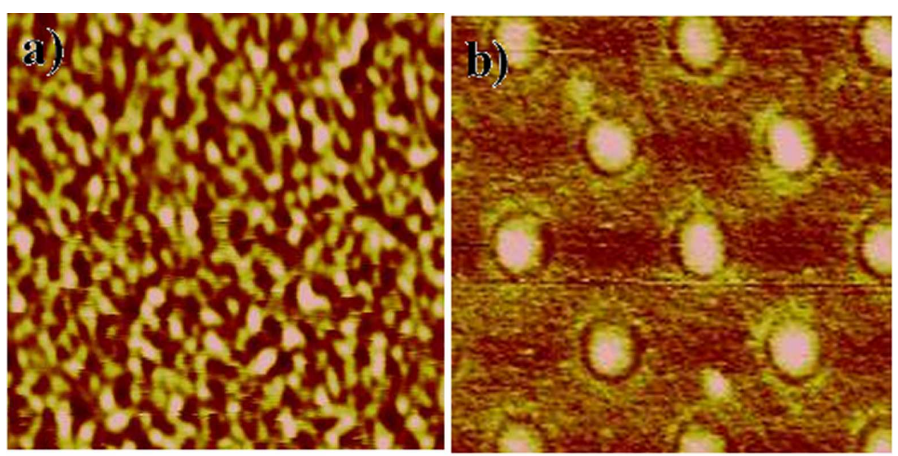

c)
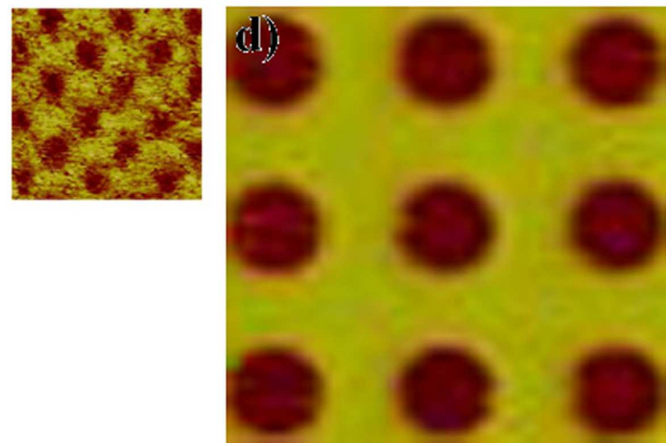

Fig. 9. MFM images of (a) non irradiated film; (b) $1 \mu \mathrm{m}$ diameter dots separated by irradiated areas (ion dose $1 \times 10^{14}$ ions $/ \mathrm{cm}^{2}$ ); (c) $250 \mathrm{~nm}$ diameter dots separated by irradiated areas (ion dose $1 \times 10^{14}$ ions $/ \mathrm{cm}^{2}$ ), (d) $1 \mu \mathrm{m}$ diameter dots separated by milled areas (ion dose $2.6 \times 10^{16}$ ions $/ \mathrm{cm}^{2}$ ).

Figure 9 shows the MFM images of the non-irradiated film and of the different patterns. The images have been acquired in the DC demagnetized state. The non-irradiated film shows an irregular domain structure. The Fourier analysis of the MFM image does not show one definite periodicity but different predominant values in the range of $250-610 \mathrm{~nm}$. The $1 \mu \mathrm{m}$ diameter hard/soft dots show a domain structure different from the film, with concentric magnetic domains reflecting the shape of their lateral confinement, as theoretically predicted [55] and observed for FePt particles with different sizes and thicknesses [30]. The $250 \mathrm{~nm}$ dots appear as single domain structures. Also the $1 \mu \mathrm{m}$ diameter milled dots do not show an evident domain structure. This suggests that the coupling with the soft matrix influences the magnetic properties of the structures. The details of such couplings deserve a further analysis.

\section{Conclusions}

By means of focused ion beam we have produced ordered arrays of submicron-sized magnetic structures. On epitaxial $\mathrm{Fe} / \mathrm{MgO}(001)$ films square submicron structures were isolated by sputtering erosion of the film. We have prelimi- 
narly studied the influence of the ion beam parameters (beam current, dwell time) on the structures shape and morphology and found the conditions for the production of sharp-edged structures. The swelling effect of the $\mathrm{MgO}$ substrate was also observed and investigated. On these structures we studied the interplay of magnetocrystalline and configurational anisotropies, showing that the overall anisotropy is not a mere superposition of the two effects and that the final anisotropy can be engineered to some extent by suitably choosing the relative orientation of the magnetocrystalline and configurational easy-axes. We then applied the ion irradiation approach to produce structures with perpendicular magnetic anisotropy on a continuous epitaxial $\mathrm{FePt} / \mathrm{MgO}(001)$ film. We found that at $\mathrm{Ga}^{+}$ion doses ranging from $1 \times 10^{14}$ to $2 \times 10^{15}$ ions $/ \mathrm{cm}^{2}$, the films undergo a complete transition from the ordered FePt $\mathrm{L}_{0}$ to the disordered A1 structure, leading to an out-of-plane to in-plane spin reorientation. Patterns of dots separated by areas irradiated with $1 \times 10^{14}$ ions $/ \mathrm{cm}^{2}$ have been compared to the same patterns obtained by sputtering erosion. We observed a coupling between the hard perpendicular dots and the soft parallel matrix, which influences the resulting domain structure.

\section{Acknowledgments}

The present work has been supported by the FIRB Project RBNE017XSW of the Italian Ministry of Education and Research, entitled "Microsystems based on magnetic materials structured on a nanoscopic scale".

\section{References}

[1] J.I. Martín, J. Nogués, Kai Liu, J.L. Vicent, Ivan K. Schuller, J. Magn. Magn. Mater 256, 449 (2003).

[2] K. Nordquist, S. Pandharkar, M. Durlam, D. Resnick, S. Teherani, D. Mancini, T. Zhu, J. Shi, J. Vac. Sci. Technol. B 15, 2274 (1997).

[3] J. Lohau, A. Moser, C.T. Rettner, M.E. Best, B.D. Terris, Appl. Phys. Lett. 78, 990 (2001).

[4] R.P. Cowburn, M.E. Welland, Science 287, 1466 (2000).

[5] A. Imre, G. Csaba, L. Ji, A. Orlov, G.H. Bernstein, W. Porod, Science 311, 205 (2006).

[6] J. Melngailis, J. Vac. Sci. Technol. B 5, 469 (1987).

[7] J. Orloff, Rev. Sci. Instrum. 64, 1105 (1993).

[8] K. Gamo, Nucl. Instrum. Methods B 121, 464 (1997).

[9] P.E. Russell, T.J. Stark, D.P. Griffis, J.R. Phillips, K.F. Jarausch, J. Vac. Sci. Technol. B 16, 2494 (1998).

[10] C. Vieu, J. Gierak, H. Launois, T. Aign, P. Meyer, J.P. Jamet, J. Ferre, C. Chappert, V. Mathet, H. Bernas, Microelectron. Eng. 53, 191 (2000).

[11] J. Gierak, H. Launois, T. Aign, P. Meyer, J.P. Jamet, J. Ferré, C. Chappert, T. Devolder, V. Mathet, H. Bernas, J. Appl. Phys. 91, 3103 (2002).

[12] J. Shen, J. Kirschner, Surf. Sci. 500, 300 (2002). 
[13] G. Xiong, D.A. Allwood, M.D. Cooke, R.P. Cowburn, Appl. Phys. Lett. 79, 3461 (2001).

[14] T. Kato, K. Suzuki, S. Tsunashima, S. Iwata, Jpn. J. Appl. Phys. 41, L1078 (2002).

[15] A.Yu. Toporov, R.M. Langford, A.K. Petford-Long, Appl. Phys. Lett. 77, 3063 (2000).

[16] P. Vavassori, D. Bisero, F. Carace, A. di Bona, G.C. Gazzadi, M. Liberati, S. Valeri, Phys. Rev. B 72, 054405 (2005).

[17] M. Albrecht, C.T. Rettner, A. Moser, M.E. Best, B.D. Terris, Appl. Phys. Lett. 81, 2875 (2002).

[18] X. Lin, J.-G. Zhu, W. Messner, IEEE Trans. Magn. 36, 2999 (2000).

[19] C. Chappert, H. Bernas, J. Ferré, V. Kottler, J.-P. Jamet, Y. Chen, E. Cambril, T. Devolder, F. Rousseaux, V. Mathet, H. Launois, Science 280, 1919 (1998).

[20] T. Devolder, C. Chappert, V. Mathet, H. Bernas, Y. Chen, J.P. Jamet, J. Ferré, J. Appl. Phys. 87, 8671 (2000).

[21] B.D. Terris, D. Weller, L. Folks, J.E.E. Baglin, A.J. Kellock, H. Rothuizen, P. Vettiger, J. Appl. Phys. 87, 7004 (2000).

[22] C. Vieu, J. Gierak, H. Launois, T. Aign, P. Meyer, J.P. Jamet, J. Ferré, C. Chappert, T. Devolder, V. Mathet, H. Bernas, J. Appl. Phys. 91, 3103 (2002).

[23] D. Raveloosona, C. Chappert, V. Mathet, H. Bernas, J. Appl. Phys. 87, 5771 (2000).

[24] T. Aoyama, I. Sato, H. Ito, S. Ishio, J. Magn. Magn. Mater. 287, 209 (2005).

[25] C.H. Lai, C.H. Yang, C.C. Chiang, Appl. Phys. Lett. 83, 4550 (2003).

[26] M. Abes, O. Ersen, D. Muller, M. Acosta, C. Ulhaq-Bouillet, A. Dinia, V. Pierron-Bohnes, Mater. Sci. Eng. C 23, 229 (2003).

[27] T. Aign, P. Meyer, S. Lemerle, J.P. Jamet, J. Ferré, V. Mathet, C. Chappert, J. Gierak, C. Vieu, Phys. Rev. Lett. 81, 5656 (1998).

[28] T. Hasegawa G.Q. Li, W. Pei, H. Saito, S. Ishio, K. Taguchi, K. Yamakawa, N. Honda, K. Ouchi, T. Aoyama, I. Sato, J. Appl. Phys. 99, 053505 (2006).

[29] A. Łapicki, E. Ahmad, T. Suzuki, J. Magn. Magn. Mater. 240, 47 (2002).

[30] Q.Y. Xu, Y. Kageyama, T. Suzuki, J. Appl. Phys. 97, $10 \mathrm{~K} 308$ (2005).

[31] H. Yamaguchi, A. Shimaze, S. Haraichi, T. Miyauchi, J. Vac. Sci. Technol. B 3, 71 (1985).

[32] F. Yongqi, N.K.A. Bryan, N.P. Hung, O.N. Shing, Rev. Sci. Instrum. 71, 1006 (2000).

[33] G.C. Gazzadi, P. Luches, S.F. Contri, A. di Bona, S. Valeri, Nucl. Instrum. Methods B 230, 512 (2005).

[34] S. Valeri, A. di Bona, P. Vavassori, in: Magnetic Properties of Laterally Confined Nanometric Structures, Ed. G. Gubbiotti, Transworld Research Network, Trivandrum (Kerala) 2006, p. 25.

[35] C. Giovanardi, A. di Bona, S. Altieri, P. Luches, M. Liberati, F. Rossi, S. Valeri, Thin Solid Films 428, 195 (2003). 
[36] E. Groppo, C. Prestipino, C. Lamberti, P. Luches, C. Giovanardi, F. Boscherini, J. Phys. Chem. B 107, 4597 (2003).

[37] A. di Bona, C. Giovanardi, S. Valeri, Surf. Sci. 498, 193 (2002).

[38] P. Luches, M. Liberati, S. Valeri, Surf. Sci. 532-535, 409 (2003).

[39] S. Benedetti, P. Luches, M. Liberati, S. Valeri, Surf. Sci. 572, L348 (2004).

[40] L. Bischoff, J. Teichert, V. Heera, Appl. Surf. Sci. 184, 372 (2001).

[41] A. Lugstein, B. Basnar, G. Hobler, E. Bertagnolli, J. Appl. Phys. 92, 4037 (2002).

[42] C. Lehrer, L. Frey, S. Petersen, H. Ryssel, J. Vac. Sci. Technol. B 19, 2533 (2001).

[43] J.F. Ziegler, J.P. Biersack, U. Littmark, The stopping and ranges of ions in solids, Pergamon, New York 1985, http://www.srim.org/.

[44] J. Nogués, T.J. Moran, D. Lederman, Ivan K. Schuller, K.V. Rao, Phys. Rev. B 59, 6984 (1999).

[45] R.P. Cowburn, A.O. Adeyeye, M. Welland, Phys. Rev. Lett. 81, 5414 (1998).

[46] J.A. Osborn, Phys. Rev. 67, 351 (1945).

[47] R.P. Cowburn, M. Welland, Appl. Phys. Lett. 72, 2041 (1998).

[48] R.P. Cowburn, M. Welland, Phys. Rev. B 58, 9217 (1998).

[49] P. Vavassori, D. Bisero, F. Carace, M. Liberati, A. di Bona, G.C. Gazzadi, S. Valeri, J. Magn. Magn. Mater. 290-291, 183 (2005).

[50] R.P. Cowburn, A. Ercole, S.J. Gray, J.A.C. Bland, J. Appl. Phys. 81, 6879 (1997).

[51] M. Grimsditch, Y. Jaccard, I.K. Schuller, Phys. Rev. B 58, 11539 (1998).

[52] R.P. Cowburn, J. Ferré, J.A.C. Bland, J. Miltat, J. Appl. Phys. 78, 7210 (1995).

[53] F. Casoli, F. Albertini, L. Pareti, S. Fabbrici, L. Nasi, C. Bocchi, R. Ciprian, IEEE Trans. Magn. 41, 3223 (2005).

[54] F. Albertini, L. Nasi, F. Casoli, S. Fabbrici, P. Luches, A. Rota, S. Valeri, J. Magn. Magn. Mater. 316, e158 (2007).

[55] S. Komineas, C.A.F. Vaz, J.A.C. Bland, N. Papanicolau, Phys. Rev. B 71, 060405(R) (2005). 